\title{
The STBUR questionnaire for predicting perioperative respiratory adverse events in children at risk for sleep-disordered breathing
}

\author{
Alan R. Tait ${ }^{1,2}$, Terri Voepel-Lewis ${ }^{1}$, Robert Christensen ${ }^{1}$ \& Louise M. O'Brien ${ }^{3,4,5}$ \\ 1 Department of Anesthesiology, University of Michigan Health System, Ann Arbor, MI, USA \\ 2 The Center for Bioethics and Social Sciences in Medicine, University of Michigan Health System, Ann Arbor, MI, USA \\ 3 Department of Neurology, University of Michigan Health System, Ann Arbor, MI, USA \\ 4 Department of Oral/Maxillofacial Surgery, University of Michigan Health System, Ann Arbor, MI, USA \\ 5 The Michael S. Aldridge Sleep Disorders Center, University of Michigan Health System, Ann Arbor, MI, USA
}

\section{Keywords}

sleep-disordered breathing; child; respiratory adverse events; perioperative

\section{Correspondence}

Alan R. Tait, Department of Anesthesiology, University of Michigan Health System, 1500 E. Medical Center Drive, Ann Arbor, MI 48109, USA

Email: atait@umich.edu

Section Editor: Charles Cote

Accepted 17 February 2013

doi:10.1111/pan.12155

\begin{abstract}
Background: In the absence of formal polysomnography (PSG), many children with symptoms of sleep-disordered breathing (SDB) go unrecognized and thus may be at risk for perioperative respiratory adverse events (PRAE). Objectives: To develop a simple practical tool to identify children with symptoms consistent with SDB who may be at risk for PRAE.

Methods: Three-hundred and thirty-seven parents of children scheduled for surgery completed the Sleep-Related Breathing Disorder (SRBD) questionnaire. Data regarding the incidence and severity of PRAE including airway obstruction and laryngospasm, were collected prospectively.

Results: Thirty-two $(9.5 \%)$ children had a confirmed diagnosis of SDB by PSG and 90 (26.7\%) had symptoms consistent with SDB based on the SRBD questionnaire. Principal component analysis identified five symptoms from the SRBD questionnaire that were strongly predictive of PRAE and which were incorporated into the STBUR tool (Snoring, Trouble Breathing, Un-Refreshed). The likelihood of PRAE was increased by threefold (positive likelihood ratio 3.06 [1.64-5.96] in the presence of any 3 STBUR symptoms and by tenfold when all five symptoms were present $(9.74$ [1.35-201.8]). In comparison, the likelihood of PRAE based on a PSG-confirmed diagnosis of SDB was 2.63 (1.17-6.23).

Conclusions: Children presenting for surgery with symptoms consistent with SDB may be at risk for PRAE. It is important therefore that anesthesia providers identify these individuals prior to surgery to avoid potential complications. The STBUR questionnaire appears promising as a simple, clinically useful tool for identifying children at risk for PRAE. Further studies to validate the STBUR questionnaire as a diagnostic tool may be warranted.
\end{abstract}

\section{Introduction}

Perioperative respiratory adverse events (PRAE) including airway obstruction, laryngospasm, and oxygen desaturation, are common sources of morbidity in children undergoing general anesthesia (1-3). Although, many of these events can be easily recognized and treated, some can be life-threatening. Children with upper respiratory tract infections (URIs), who are obese and undergo surgery involving the airway (1-4), or who have sleep-disordered breathing (SDB) may be particularly vulnerable to PRAE (5-8).

Sleep-disordered breathing represents a spectrum of breathing disturbances ranging from snoring to obstructive sleep apnea (OSA). Approximately, 10-12\% of school-aged children have some type of SDB $(9,10)$ with 
symptoms varying from simple sleep disturbances to hypopnea and apnea (11). Furthermore, childhood SDB can lead to neurocognitive and behavioral changes, cardiovascular disease, and poor school performance $(12,13)$. Importantly, SDB has been associated with an increased risk for PRAE including airway obstruction, oxygen desaturation, and the need for intervention (5-8). Unfortunately, in the absence of formal testing, many 'at risk' children may go unrecognized preoperatively (11). Indeed, Sinha et al. reported on two cases of perioperative apnea in children with undiagnosed SDB (14). It is thus important that children who have symptoms (mild and overt) which are consistent with SDB are identified preoperatively.

Although PSG is considered the 'gold standard' for diagnosing SDB (15), routine preoperative use is neither practical nor economical. As a pragmatic measure, many anesthesia providers ask parents whether their child snores, has sleep apnea, or has ever been diagnosed with SDB. This is consistent with the findings of Parnis et al. who showed that children who snore are at greater risk of PRAE (1). However, in practice, the nature and consistency of these questions may vary between institutions and providers.

Adopting a more standardized approach to the preoperative evaluation of 'at risk' children, the American Society of Anesthesiologists (ASA) published guidelines for the management of patients with OSA and recommended a checklist for routine screening (16). Unfortunately, medical history and physical examination have been shown to be unreliable for diagnosing OSA (17). Furthermore, this checklist has only been validated in adults (18) and, to our knowledge, its ability to identify children at risk for PRAE has not been explored. Abrishami et al. however, note that questionnaires '... may facilitate early detection of patients who need further assessment and who would benefit from perioperative precautions for OSA patients' (19). This study therefore was designed to develop a simple and practical screening tool for identifying children with symptoms consistent with SDB who may be at risk for developing PRAE.

\section{Methods}

This study was approved by the University of Michigan's Institutional Review Board with written informed consent. Children aged 2-14 years presenting for elective surgery requiring general anesthesia were included. Exclusion criteria include ASA class 3-5, emergency surgery, history of cardiovascular disease, or procedures requiring total intravenous anesthesia. Anesthesia management was at the discretion of the anesthesia provider.
To identify children 'at risk' for SDB, research assistants surveyed all parents using the Sleep-Related Breathing Disorder (SRBD) questionnaire (20). Surveys were completed by hand prior to or during their child's surgery. The SRBD is a subscale of the Pediatric Sleep Questionnaire which has been validated for SDB screening in children 2-18 years of age (20). This tool also contains two validated four-item subscales both of which have been used independently to evaluate snoring and daytime sleepiness (20-24). Response options are 'yes', 'no', and 'don't know'. Scores for all scales and subscales are generated by the number of positive responses out of the number answered. A child with a score of $\geq 0.33$ on either the SRBD or its two 4-item subscales is considered at risk for SDB, daytime sleepiness, and snoring, respectively (20).

\section{Outcome measures}

Data were collected prospectively by trained research assistants and included demographics (age, gender, race/ ethnicity), type and duration of anesthesia and surgery, and comorbidities (e.g., asthma, obesity, and URI). The records of all children were reviewed to see whether PSG had been performed. Data regarding the incidence and severity of PRAE were documented on data collection sheets at induction including airway device placement, emergence including airway device removal, and recovery in the Postoperative Care Unit (PACU) by the anesthesia provider. The definitions of PRAE and methods of data collection have been described previously (see Table 1) (4). For the purpose of this study, the primary outcome was the appearance of at least one critical PRAE during the perioperative period (induction through PACU discharge: average 3.08 h) (4).

\section{Statistical analysis and Sample size}

Statistical analyses were performed using PASW software (v18.0; PASW Inc., Chicago, IL, USA). Nonparametric data were analyzed using Mann-Whitney U-test, chi-square test, and Fisher's exact test. Parametric data were analyzed using unpaired $t$-tests. All predictive parameters were calculated including sensitivity and specificity, positive and negative predictive values (PPV and NPV), positive and negative likelihood ratios (LR+ and $\mathrm{LR}-$ ), and odds ratios (OR) with $95 \%$ confidence intervals. Greater reliance was placed on the LR+ and LR - values because they express the increasing or decreasing likelihood of an outcome at the individual patient level $(25,26)$. Receiver operator characteristic (ROC) curves were generated and the area under the curve (AUC or $c$ statistic) calculated, where appropriate. 
Table 1 Definitions of airway events

\begin{tabular}{|c|c|}
\hline \multicolumn{2}{|l|}{ Oxygen desaturation } \\
\hline Minor & $\begin{array}{l}\text { 5-10\% decrease from baseline } \\
\text { (preinduction value) }\end{array}$ \\
\hline Major & $>10 \%$ decrease from baseline \\
\hline \multicolumn{2}{|l|}{ Coughing } \\
\hline Minor & One to several coughs \\
\hline Major & Continuous coughing \\
\hline \multicolumn{2}{|l|}{ Breath Holding } \\
\hline Minor & $<30 \mathrm{~s}$ \\
\hline Major & $>30 \mathrm{~s}$ requiring an intervention \\
\hline \multicolumn{2}{|l|}{ Airway obstruction } \\
\hline Minor & $\begin{array}{l}\text { Corrected with repositioning or placement } \\
\text { of an oral/nasal airway }\end{array}$ \\
\hline Major & $\begin{array}{l}\text { Requiring jaw-chin thrust maneuver or } \\
\text { placement of a laryngeal mask airway or ETT }\end{array}$ \\
\hline Laryngospasm & $\begin{array}{l}\text { Requiring continuous positive airway } \\
\text { pressure or intervention with a muscle } \\
\text { relaxant }\end{array}$ \\
\hline Bronchospasm & Auscultated wheezing \\
\hline $\begin{array}{l}\text { Minor adverse } \\
\text { respiratory event }\end{array}$ & $\begin{array}{l}\text { At least one adverse respiratory event during } \\
\text { the perioperative period }\end{array}$ \\
\hline $\begin{array}{l}\text { Critical respiratory } \\
\text { adverse event }\end{array}$ & $\begin{array}{l}\text { At least one major adverse respiratory } \\
\text { event (can also include any episode of } \\
\text { laryngospasm or bronchospasm) during } \\
\text { the perioperative period (induction through } \\
\text { discharge from PACU) }\end{array}$ \\
\hline
\end{tabular}

ETT, endotracheal tube; PACU, postanesthesia care unit. Reproduced with permission: Tait et al (4).

A principal factor analysis with varimax rotation was performed to identify underlying factors in the SRBD questionnaire. Items in the questionnaire were deemed to define a given component if the loading factor was $>0.50$. Criteria used to determine the number of factors to be retained were based on accepted decision rules (27). Reliability of items in the questionnaire was measured using Cronbach's alpha with values of $\geq 0.7$ reflecting excellent reliability.

Data from this study were part of a large prospective dataset examining PRAE and postoperative behaviors in children with symptoms of SDB. As such, the initial sample size was based on a primary outcome of adverse events. For the purposes of this study, however, the sample size more than satisfies the rule of thumb for instrument development of 10 subjects per item in a questionnaire $(28,29)$.

\section{Results}

A total of 439 eligible parents were approached to participate. Of these, 77 declined and 25 were excluded due to the following: surveys not completed (9), no general anesthesia (9), did not meet inclusion criteria (5), and surgery canceled (2). Three hundred thirty- seven completed questionnaires were received; however, complete outcome data (overall critical PRAE) were only available for 302 . The demographics of the participants are described in Table 2. Thirty-two children $(9.5 \%)$ underwent a preoperative PSG, and all were positive for OSA. Ninety $(26.7 \%)$ children met the criteria for high risk SDB as defined by the SRBD questionnaire. As expected, significantly more children with symptoms consistent with SDB underwent tonsillectomy/adenoidectomy ( $\mathrm{T} \& \mathrm{~A}$ ) surgery compared with children without SDB risk $(58.9 \%$ vs $4.5 \%$, $P<0.001)$. There were no differences in acute URI status $(0 \%$ vs $0.8 \%)$, sevoflurane use $(97.8 \%$ vs $92.7 \%$ ), and opioid usage (perioperative morphine equivalents $/ \mathrm{kg} / \mathrm{hr}$ [per standard conversion formulae $=0.17$ vs 0.14 ), respectively between those with and without SDB-like symptoms.

Given that the SRBD tool has been validated in children for SDB in the clinical research setting, we were interested in examining whether specific items in the questionnaire were able to predict PRAEs in children in the surgical setting. As such, we first examined the pre-

Table 2 Demographics

\begin{tabular}{|c|c|}
\hline & $N=337$ \\
\hline Gender (M/F\%) & $53 / 47$ \\
\hline Age (years) & $6.58 \pm 3.36$ \\
\hline \multicolumn{2}{|l|}{ Race } \\
\hline Caucasian & 276 (81.9) \\
\hline Black & $24(7.1)$ \\
\hline Hispanic & $13(3.9)$ \\
\hline ASA status (I/II\%) & $46 / 54$ \\
\hline \multicolumn{2}{|l|}{ Surgical service } \\
\hline Urology & $50(14.8)$ \\
\hline General Surgery & $59(17.5)$ \\
\hline ENT-T \& A & $64(19.0)$ \\
\hline ENT-non-T \& A & $44(13.1)$ \\
\hline Orthopaedics & $48(14.2)$ \\
\hline Ophthalmology & $32(9.5)$ \\
\hline Other & $40(11.9)$ \\
\hline Anesthesia duration (mins) & $68.7 \pm 59.8$ \\
\hline Surgery duration (mins) & $48.7 \pm 52.3$ \\
\hline PACU duration (mins) & $116.7 \pm 61.6$ \\
\hline \multicolumn{2}{|l|}{ Medical history } \\
\hline Asthma & 47 (13.9) \\
\hline Obesity $^{a}$ & $46(13.1)$ \\
\hline URI active ${ }^{\mathrm{b}}$ & $2(0.6)$ \\
\hline URI recent (within 4 weeks) ${ }^{b}$ & $10(3.0)$ \\
\hline
\end{tabular}

Data presented as $n(\%)$ or mean $\pm S D$

ENT, otolaryngology; T \& A, adenotonsillectomy; ASA, American Society of Anesthesiologists physical status; URI, upper respiratory infection; PACU, postanesthesia care unit.

${ }^{a}$ Obesity based on age and gender adjusted percentiles.

${ }^{\mathrm{b}}$ Per parent report and preoperative history and physical. 
dictive parameters of the two validated four-item SRBD subscales ('snoring' and 'sleepiness'). The snoring subscale revealed a LR+ of $1.71(1.18-2.52)$ and OR of 2.17 (1.27-3.71, $P=0.002)$. The PPV and NPV were $66.3 \%$ and $52.4 \%$, respectively. The sleepiness subscale produced a LR+ of $1.54(1.02-2.36)$ and an OR of 1.79 (1.03-3.14, $P=0.036)$. The PPV and NPV were $63.7 \%$ and $50.5 \%$, respectively. These data suggested that the four-item 'snoring' subscale had similar predictive parameters compared with the parent SRBD questionnaire and thus would be easier to administer in the clinical setting.

Adopting another approach to reducing the number of items in the SRBD questionnaire, we performed a factor analysis of all items. This analysis identified 11 items which accounted for $32.6 \%$ of the variance in the model. Next, the predictive values for PRAE of each of the 11 individual items identified in the factor analysis were examined, and the five with the highest values were selected. This resultant five-item questionnaire was named the STBUR scale (Snoring, Trouble Breathing, and Un-Refreshed, Table 3).

The ability of the STBUR to identify children 'at risk' for SDB was evaluated against the SRBD questionnaire $(n=337)$ and PSG $(n=32)$ using ROC curves. Analysis indicated excellent ability of these tools to identify those at risk for $\mathrm{SDB}$, that is, $c$ statistic $(95 \% \mathrm{CI})=0.97(0.95-0.99), 0.88(0.84-0.93)$, and 0.83 (0.76-0.91), respectively. Table 4 compares the predictive parameters by the different screening methods and shows that the likelihood of a critical PRAE was increased threefold in the presence of any three STBUR symptoms and by tenfold when all symptoms were present. These results compare very favorably with the 1.9-fold likelihood of a PRAE when using the SRBD questionnaire and a 2.6-fold likelihood of PRAE in children with a PSG-confirmed diagnosis of SDB. Given that more children with SDB symptoms underwent $\mathrm{T} \& \mathrm{~A}$, we examined the ability of the STBUR to predict PRAE for other types of surgery. Results showed that the positive likelihood of a critical PRAE was increased by fourfold $(4.21,1.15-18.6)$ among chil-

Table 3 Symptom items comprising the STBUR questionnaire

While sleeping, does your child...

1.... snore more than half the time?

2. ... snore loudly?

3. ... have trouble breathing, or struggle to breathe?

4. Have you ever seen your child stop breathing during the night?

5. Does your child wake up feeling unrefreshed in the morning?

STBUR, Snoring, Trouble Breathing, Un-Refreshed. dren presenting for non-T \& A surgery with any three STBUR symptoms.

The majority of PRAEs occurred in children undergoing $\mathrm{T} \& \mathrm{~A}$ compared with non-T \& A surgery $(71.4 \%$ vs $48.5 \%, P=0.002)$ and during removal of an airway device $(15.7 \%, n=286)$ and in the PACU $(36.1 \%$, $n=335)$. The majority of PACU events were arterial oxygen desaturations. Twenty-eight children $(8.6 \%$, $n=326)$ had critical PRAEs on induction of anesthesia and $25(8.5 \%, n=293)$ during airway device placement. Table 5 compares the incidences of individual events based on the STBUR criteria with those of a PSG-confirmed diagnosis of SDB. Results show the ability of the STBUR questionnaire to identify children at risk for specific PRAEs and confirm the findings of others $(5-7,14,30)$ that children with SDB-associated symptoms are at greater risk of PRAE compared with those without.

\section{Discussion}

Results showed that a significant proportion of children presenting for surgery had symptoms consistent with SDB. Although some children presenting for T \& A will have undergone a preoperative work-up for SDB, including PSG, and are thus considered 'at risk', many children are not evaluated and, as such, may present with unrecognized risk potential. Indeed, our study confirmed previous findings that children with symptoms consistent with SDB are at increased risk for perioperative complications $(5-8,14)$ and, as such, reinforces the importance of identifying those at risk for SDB prior to surgery.

This study describes the development of the STBUR questionnaire for identifying children with symptoms consistent with SDB who may be at risk for PRAE. We should emphasize that although many of the children in our study may indeed have had underlying SDB, not all underwent confirmatory formal PSG. However, although further validation is necessary to determine whether the STBUR questionnaire can be used as a diagnostic tool, it clearly shows a significant relationship with PRAE outcomes. Given the impracticality of obtaining PSG on all children, and in the absence of reliable clinical indicators, Brietzke et al (17). suggest the need for 'A simple, inexpensive screening test than can be linked to SDB outcomes ....' To this end, the STBUR appears to satisfy this need in its simplicity and ability to identify children at risk for PRAE. Although none of the PRAEs in this study resulted in serious morbidity, the ability to anticipate, recognize, and treat adverse events is nevertheless paramount (31). 
Table 4 Predictive parameters for PRAE by screening method

\begin{tabular}{lllcc}
\hline & SRBD & PSG & Three-item STBUR \\
$n=84 / 302^{\mathrm{a}}$ & $n=32 / 302^{\mathrm{a}}$ & $54 / 298^{\mathrm{a}}$ & $\begin{array}{c}\text { Five-item STBUR } \\
n=12 / 296^{\mathrm{a}}\end{array}$ \\
\hline Sensitivity (\%) & $35.4(30.4-39.9)$ & $14.9(11.4-17.4)$ & $26.4(22.2-29.6)$ & $7.00(4.60-7.60)$ \\
Specificity (\%) & $80.9(75.1-86.0)$ & $94.3(90.3-97.2)$ & $91.4(86.5-95.0)$ & $99.3(96.6-1.00)$ \\
PPV (\%) & $67.9(58.3-76.5)$ & $75.0(57.2-87.7)$ & $77.8(65.3-87.2)$ & $91.7(60.4-99.6)$ \\
NPV (\%) & $52.3(48.6-55.6)$ & $49.3(47.2-50.8)$ & $52.0(49.3-54.1)$ & $48.6(47.3-48.9)$ \\
LR+ & $1.85(1.23-2.85)$ & $2.63(1.17-6.23)$ & $3.06(1.64-5.96)$ & $9.74(1.35-201.8)$ \\
LR- & $0.79(0.70-0.93)$ & $0.90(0.85-0.98)$ & $0.81(0.74-0.90)$ & $0.94(0.92-0.98)$ \\
Odds ratio & $2.32(1.32-4.07)^{\mathrm{b}}$ & $2.91(1.17-6.90)^{\mathrm{b}}$ & $3.80(1.83-8.04)^{\mathrm{b}}$ & $10.40(1.37-218.3)^{\mathrm{b}}$ \\
\hline
\end{tabular}

STBUR, Snoring, Trouble Breathing, Un-Refreshed; PRAE, perioperative respiratory adverse events; SRBD, sleep-related breathing disorder questionnaire; PSG, polysomnography; PPV, positive predictive value; NPV, negative predictive value; LR+, positive likelihood Ratio; LR-, negative likelihood Ratio.

${ }^{a} n=$ number with the characteristic/N.

${ }^{\mathrm{b}} \mathrm{P}<0.01$.

Table 5 Individual perioperative respiratory adverse events (PRAE) in children with and without SDB-associated symptoms

\begin{tabular}{lccc}
\hline & $\begin{array}{l}\text { No SDB } \\
\text { Risk }^{\mathrm{a}} \\
n=218\end{array}$ & $\begin{array}{l}\text { STBUR: Any } \\
\text { three items } \\
n=54\end{array}$ & $\begin{array}{l}\text { PSG } \\
n=32\end{array}$ \\
\hline Major cough & $30(14.0)$ & $15(27.8)$ & $9(27.3)$ \\
Major breath hold & $1(0.5)$ & $3(5.7)$ & $3(9.4)$ \\
Laryngospasm & $8(3.8)$ & $6(11.3)$ & $3(9.1)$ \\
Bronchospasm & $1(0.5)$ & $2(3.8)$ & $2(6.1)$ \\
Airway obstruction & $47(21.5)$ & $27(50.0)$ & $15(45.5)$ \\
Major desaturation & $70(34.5)$ & $28(52.8)$ & $21(61.8)$ \\
Critical PRAE $^{\text {b }}$ & $104(47.7)$ & $42(77.8)$ & $24(75.0)$ \\
\hline
\end{tabular}

Data are presented as $n(\%)$.

PSG, polysomnography; PRAE, perioperative respiratory adverse event; PACU, postanesthesia care unit; STBUR, Snoring, Trouble Breathing, Un-Refreshed; SDB, sleep-disordered breathing

${ }^{a}$ No SDB risk based on SRBD questionnaire (i.e., score $<0.33$ ).

${ }^{b}$ At least one major adverse respiratory event (can also include the occurrence of any laryngospasm or bronchospasm) during the perioperative period (induction through discharge from the PACU).

Despite the fact that there are several tools available for detecting OSA in adults (e.g., the Berlin, STOP, and ASA questionnaires), none have been validated in children $(16,18,32,33)$. Although there are elements of these questionnaires that are pertinent to children (e.g., snoring and sleepiness), these instruments also include adult cut points or definitions for BMI, neck circumference, and blood pressure that are not valid for children $(16,18,32,33)$. Therefore, given the paucity of clinically validated SDB-screening tools for children, we chose the pediatric SRBD tool developed by Chervin et al. as the basis for this study. Although this tool has only been validated in clinical research settings, it associated strongly with PSG-confirmed SDB leading the authors to suggest that in the absence of PSG, the SRBD is a valid and reliable instrument to screen for SDB in both nonsurgical children and those prior to and following T \& A $(20,23,24)$. Furthermore, in an evidence-based review of subjective pediatric sleep measures, the Pediatric Sleep Questionnaire and its subscale SRBD were shown to have excellent psychometric reliability and validity and met 'well established' evidence-based assessment criteria (21). Indeed, our results showed that selected items from the SRBD questionnaire were strongly predictive of PRAE in our population of children and performed well in the subset of 32 children who underwent PSG.

Because of the narrow window of opportunity to assess children during the preoperative exam, any screening tool must be simple to use. With this in mind, we performed data reduction techniques on items in the SRBD to identify salient factors which could be used to further refine our tool. Consistent with many other SDB-screening tools, these factors consisted of a snoring, breathing, and sleepiness component (20). Results showed that the STBUR questionnaire performed favorably against the parent SRBD and, more importantly, against the 'gold standard' PSG in identifying children at risk for SDB and PRAE.

Potential limitations of the study are acknowledged. First, as stated, without further validation against PSG, the STBUR tool should not be considered diagnostic for SDB but only as a tool for identifying children with symptoms consistent with SDB who are at risk for PRAE. Second, although previously published, we recognize that our definitions of PRAE may be open to debate. As such, the generalizability of our results should be interpreted within the context of these definitions. Third, although the STBUR tool appears to be excellent in predicting PRAE in otherwise healthy children with SDB-associated symptoms, the ability to 
predict other types of complications or to predict PRAEs in children with comorbidities has yet to be determined. Validation in different populations and using different outcome measures may therefore be warranted.

Results of this study showed that there may be a significant number of children who present for surgery with unrecognized SDB-associated symptoms and who may be at risk for PRAE. It is imperative therefore that anesthesia providers are able to recognize the child 'at risk', anticipate potential problems and, if necessary, modify their anesthetic management to optimize care (34). The development of the STBUR questionnaire appears significant in its ability to identify children with symptoms consistent with SDB who are at risk for PRAE. Furthermore, from a pragmatic perspective, it is simple, easy to use and thus would likely have excellent clinical utility in a busy preoperative anesthesia setting. Although the underlying assumption is that the STBUR tool may be able to identify some children with undiagnosed SDB, further studies will be necessary to validate this potentially important and clinically relevant tool against PSG and in different settings.

\section{Acknowledgments}

The authors are indebted to Maureen McGonegal B.A., Jennifer Hemberg, B.S., Lauren Perlin, B.S., Elsa Pechlivanidis, B.S., B.S.N., Bianca Lawrence, and Katherine Lypen for help with subject recruitment and data collection.

\section{Disclosures}

This study was approved by the University of Michigan's Institutional Review Board. There are no conflicts of interest to report.

\section{Financial support}

This study was supported, in part, by the Department of Anesthesiology, University of Michigan Health System. Dr. Tait is also supported by a grant from the National Institutes of Health, Bethesda MD (NICHD: R01H D053594), as is Dr. O'Brien (NHLBI: K23HL095739, R21 HL87819, and R21HL089918).

\section{Conflict of interest}

No conflicts of interest declared.

\section{References}

1 Parnis SJ, Barker DS, Van Der Walt JH Clinical predictors of anaesthetic complications in children with respiratory tract infections. Paediatr Anaesth 2001; 11: 29-40.

2 von Ungern-Sternberg B, Boda K, Chambers $\mathrm{N}$ et al. Risk assessment for respiratory complications in paediatric anaesthesia: a prospective cohort study. Lancet 2010; 376: 773-783.

3 Tait AR, Malviya S, Voepel-Lewis T et al. Risk factors for perioperative adverse respiratory events in children with upper respiratory tract infections. Anesthesiology 2001; 95: 299-306.

4 Tait AR, Voepel-Lewis T, Kostrzewa A et al. Incidence and risk factors for perioperative adverse events in children who are obese. Anesthesiology 2008; 108: 375-380.

5 Hwang D, Shakir N, Limann B et al. Association of sleep-disordered breathing with postoperative complications. Chest 2008; 133: 1128-1134.

6 Raghavendran S, Bagry H, Detheux G et al. An anesthetic management protocol to decrease respiratory complications after adenotonsillectomy in children with severe sleep apnea. Anesth Analg 2010; 110: 1093-1101.

7 Sanders J, King M, Mitchell R et al. Perioperative complications of adenotonsillectomy in children with obstructive sleep apnea syndrome. Anesth Analg 2006; 103: 1115-1121.

8 Waters K, McBrien F, Stewart P et al. Effects of OSA, inhalational anesthesia, and fentanyl on the airway and ventilation of children. J Appl Physiol 2002; 92: $1987-$ 1994.

9 Ali N, Pitson D, Stradling J. Snoring, sleep disturbance, and behaviour in 4-5 year olds. Arch Dis Child 1993; 68: 360-366.

10 Lumeng J, Chervin R. Epidemiology of pediatric obstructive sleep apnea. Proc Am Thorac Soc 2008; 5: 242-252.

11 Brown K. Outcome, risk, and error and the child with obstructive sleep apnea. Pediatr Anesth 2011; 21: 771-780.

12 Zhao Q, Sherrill D, Goodwin J et al. Association between sleep disordered breathing and behavior in school-aged children: the Tuscon children's assessment of sleep apnea. Open Epidemiol J 2008; 1: 1-9.

13 O'Brien LM. Neurocognitive and behavioral consequences of sleep disordered breathing in children. In: Ivanenko A, ed. Sleep and Psychiatric Disorders in Children and Adolescents. New York: Informa Healthcare, Inc., 2008:149-161.

14 Sinha R, Bose S, Thangaswamy C. Unrecognized obstructive sleep apnea in children: caught on the wrong foot and lessons learnt!!!. Pediatr Anesth 2008; 18: 984-986.

15 American Academy of Pediatrics. Section of Pediatric Pulmonology, Subcommittee on obstructive sleep apnea syndrome. Clinical practice guideline: diagnosis and management of childhood sleep apnea syndrome. Pediatrics 2002; 109: 704-712.
16 Gross J, Bachenberg K, Benumof J et al. Practice guidelines for the perioperative management of patients with obstructive sleep apnea: a report by the American Society of Anesthesiologists task force on perioperative management of patients with obstructive sleep apnea. Anesthesiology 2006; 104: 1081-1093.

17 Brietzke S, Katz E, Roberson D. Can history and physical examination reliably diagnose obstructive sleep apnea/hypopnea syndrome? A systematic review of the literature. Otolaryngol Head Neck Surg 2004; 131: 827-832.

18 Chung F, Yegneswaran B, Liao P et al. Validation of the Berlin Questionnaire and American Society of Anesthesiologists checklist as screening tools for obstructive sleep apnea in surgical patients. Anesthesiology 2008; 108: 822-830.

19 Abrishami A, Khajehdehi A, Chung F. A systematic review of screening questionnaires for obstructive sleep apnea. Can J Anesth 2010; 57: 423-438

20 Chervin R, Hedger K, Dillon J et al. Pediatric sleep questionnaire (PSQ): validity and reliability of scales for sleep-disordered breathing, snoring, sleepiness, and behavioral problems. Sleep Med 2000; 1: 21-32.

21 Lewandowski A, Toliver-Sokol M, Palermo T. Evidence-based review of subjective pediatric sleep measures. J Pediatr Psychol 2011; 36: 780-793. 
22 O'Brien LM, Lucas N, Felt B et al. Aggressive behavior, bullying, snoring, and sleepiness in schoolchildren. Sleep Med 2011; 12: 652-658.

23 Chervin R, Weatherly R, Ruzicka D et al. Subjective sleepiness and polysomnographic correlates in children scheduled for adenotonsillectomy vs other surgical care. Sleep 2006; 29: 495-503.

24 Weatherly R, Ruzicka D, Marriott D et al. Polysomnography in children scheduled for adenotonsillectomy. Otolaryngol Head Neck Surg 2004; 131: 727-731.

25 Attia J. Moving beyond sensitivity and specificity: using likelihood ratios to help interpret diagnostic tests. Aust Prescr 2003; 26: 111-113.
26 Nofuentes J. de Dios Luna del Castillo J. Comparison of the likelihood ratios of two binary diagnostic tests in paired designs. Stat Med 2007; 26: 4179-4201.

27 Thompson B, Daniel G. Factor analyticevidence for construct validity scores: a historical overview and some guidelines. Educ Psychol Meas 1996; 56: 197-208.

28 Arrindell W, van der Ende J. An empirical test of the utility of the observations-to-variables ratio in factor and component analysis. Appl Psychol Meas 1985; 9: 165-178.

29 Velicer W, Fava J. Effects of variable and subject sampling on factor pattern recovery. Psychol Methods 1998; 3: 231-251.

30 Rosen G, Muckle R, Mahowald M et al. Postoperative respiratory compromise in children with obstructive sleep apnea syndrome: can it be anticipated. Pediatrics 1994; 93: $784-88$

31 Coté CJ. The upper respiratory tract infection (URI) dilemma: fear of complication or litigation? Anesthesiology 2001; 95: 283-285.

32 Chung F, Yegneswaran B, Liao P et al. STOP questionnaire: a tool to screen patients for obstructive sleep apnea. Anesthesiology 2008; 108: 812-821.

33 Netzer N, Stoohs R, Netzer C et al. Using the Berlin questionnaire to identify patients at risk of having sleep apnea syndrome. Ann Intern Med 1999; 131: 485-491.

34 Lerman J. Unraveling the mysteries of sleep-disordered breathing in children. Anesthesiology 2006; 105: 645-646. 